\title{
Direct PtSn Alloy Formation by Pt Electrodeposition on Sn Surface
}

\author{
Jan N. Schwämmlein, Paulette A. Loichet Torres, Hubert A. Gasteiger \& Hany A. El-Sayed \\ Electrochemical deposition is a viable approach to develop novel catalyst structures, such as $\mathrm{Pt}$ thin \\ films on conductive support materials. Most studies, reaching out to control electrochemical deposition \\ of Pt to monolayer quantities focus on noble metal substrates (e.g., Au). In contrast, conductive oxides, \\ such as antimony doped tin oxide (ATO), are considered as support material for different applications, \\ e.g., as fuel cell catalysts. Herein, we investigate the deposition process of Pt on Sn, used as a model \\ system for the electrochemical deposition of Pt on non-noble metal oxide supports. Doing so, we shade \\ some light on the differences of a metallic Sn surface and surface oxide species in electrochemical \\ deposition processes. With respect to a borate buffer solution, containing $\mathrm{K}_{2} \mathrm{PtCl}_{4}$ as $\mathrm{Pt}$ precursor, \\ we report for the first time that surface oxides have the capability to fully inhibit the electrochemical \\ deposition of Pt. Furthermore, direct alloying of the deposited Pt with the Sn support during the \\ electrodeposition process yielded a catalyst with a high activity for the oxidation of $C O$.
}

Fuel cells are considered as a candidate to replace the currently wide-spread combustion engine and limit the exhaust of $\mathrm{CO}_{2}$, e.g., caused by automotive traffic. Especially since first car producers, such as Toyota ${ }^{1}, \mathrm{Hyundai}^{2}$, and $\mathrm{Honda}^{3}$, finally brought fuel cell powered vehicles to the market, this technology is in reach of widespread application. Nevertheless, there is a demand for novel electrocatalysts due to the low abundance of Pt and the limited stability of current catalysts under fuel cell operating conditions ${ }^{4}$. In this respect, film-like structures on non-noble metal based supports, such as antimony doped tin oxide (ATO), are of potential interest due to various reasons. First of all, the Pt surface to mass ratio of extended surfaces is similar to that of nanoparticles for sufficiently thin layers. Second, Pt thin film structures may show high specific activity towards the ORR due to a likely exposure of low index facets, providing a superior ORR exchange current density compared to Pt nanoparticles $^{5}$. Third, a contiguous film may protect the underlying support material against corrosion. Finally, conductive oxides were proposed as stable catalysts with respect to high anodic potentials, occurring under certain fuel cell operating conditions, such as start-up or shut-down (SUSD) $)^{6-8}$

In contrast to the lack of synthesis methods for Pt thin films on non-noble metal oxides, various methods were already presented to generate low loading platinum deposits on metallic supports. One approach was to utilize the redox replacement of an underpotentially adsorbed $\mathrm{Cu}$ layer by Pt from a precursor dissolved in the electrolyte ${ }^{9}$. Another method, developed by Brimaud et al. utilizes the strong adsorption of CO on the deposited Pt to hinder any further deposition after completion of a single Pt monolayer (ML) on a $\mathrm{Au}(111)$ single $\mathrm{crystal}^{10}$. Though the $\mathrm{Pt}$ ML was reported to be stable while CO was adsorbed, the group showed that the structure collapsed to Pt islands as soon as CO was displaced. A similar effect to limit Pt electrodeposition on $\mathrm{Au}(111)$ to an amount of Pt equivalent to one ML was presented by Moffat and co-workers ${ }^{11}$. Instead of CO, the potential dependent formation of $\mathrm{H}_{\mathrm{ads}}$ was used to interrupt the deposition process while the hydrogen evolution reaction (HER) proceeded on the Pt deposit. To avoid the formation of $\mathrm{H}_{2}$ bubbles, the group used an unbuffered electrolyte with a $\mathrm{pH}$ of 4 , where the amount of HER was limited due to an evolving $\mathrm{pH}$ gradient between the electrode and the bulk of the electrolyte $^{12}$. The scanning tunneling microscopy (STM) images of the resulting deposits showed an island-like morphology of very small Pt deposits on the Au surface. Similar to those studies, most electrochemical deposition techniques, aiming to achieve low loading Pt deposits focus on noble metal substrates, whereas deposition of low platinum quantities on non-noble metals or conductive oxides was only scarcely reported (e.g., on $\mathrm{Ni})^{13}$.

In this publication, we present insights into the electrodeposition process of $\mathrm{Pt}$ on $\mathrm{Sn}$ as a model system for such novel catalysts. Overall, this manuscript aims to deliver a better understanding of the electrodeposition process, eventually leading to a synthesis approach for novel fuel cell catalysts, comprised of low Pt loaded conductive oxides. At first, the electrochemical behavior of $\mathrm{Sn}$ in slightly alkaline solution is investigated to identify the oxidation and reduction processes on its surface. Subsequently, the influence of oxides on the electrochemical 
deposition process of Pt on the Sn surface is evaluated. Finally, Pt deposition is carried out in the presence of HER, where the resulting Pt film thickness is studied by energy dispersive X-ray (EDX) spectroscopy. The electrochemical oxidation of $\mathrm{CO}$ is used as a probe for the structure of the deposits. By this method, we are able to identify the deposits as PtSn alloys, formed directly on the surface of the electrode during the deposition process.

\section{Methods}

Rotating disk electrode (RDE) setup. A home-made glass cell was used for all electrochemical measurements presented here. All compartments of the cell were thoroughly cleaned in Caro's acid and heated repetitively in fresh, ultrapure water $(18.2 \mathrm{M} \Omega \mathrm{cm}$, MilliQ Ingegral, Merck Millipore KGaA, Germany) to eliminate sources of contamination. Borate buffer $(0.05 \mathrm{M})$ was prepared from high purity $\mathrm{H}_{3} \mathrm{BO}_{3}(99.97 \%$, trace metals basis, Sigma Aldrich Corp., Germany) and $\mathrm{NaOH} \cdot \mathrm{H}_{2} \mathrm{O}$ (99.9995\%, metals basis, TraceSELECT, Sigma Aldrich Corp., Germany) by addition of ultrapure water. The $\mathrm{pH}$ was measured with a $\mathrm{pH}$ meter by separating a small portion of the electrolyte and subsequently adding sodium hydroxide or boric acid to the stock solution until the desired value of 8.4 was reached. Argon used to saturate the electrolyte was of high purity (6.0 grade, Westfalen $A G$, Germany), as well as carbon monoxide (4.7-grade, Westfalen AG, Germany) for CO oxidation voltammetry. A home-made Ag/AgCl reference electrode, saturated with $\mathrm{KCl}$ (99.999\%, Sigma Aldrich Corp., Germany) was used for all measurements. The reference potential of the $\mathrm{Ag} / \mathrm{AgCl}$ electrode was calibrated with the platinum ring of the electrode by saturating the electrolyte with $\mathrm{H}_{2}$ prior to the performed experiment. The reference electrode was separated from the cell compartment by an electrolyte bridge to avoid contamination of the electrolyte with chloride ions. All potentials in this publication are reported vs the reversible hydrogen electrode (RHE) potential, calculated by subtraction of the reference electrode potential from the measured value. All area-normalized currents refer to the geometric area of the electrode (e.g., $\mathrm{cm}^{2}$ ), if not otherwise stated. Electrochemical measurements were performed using an Autolab potentiostat (PGSTAT302N, Metrohm AG, Switzerland) and a rotator (Pine Research Instrumentation, USA) with a polyether ether ketone shaft.

Electrode preparation. Polycrystalline Sn electrodes (99.999\%, MaTecK Material-Technologie \& Kristalle $\mathrm{GmbH}$, Germany) were prepared by polishing in three individual steps using a $9 \mu \mathrm{m}$ diamond suspension (MetaDi Supreme) on a VerduTex polishing cloth, a $3 \mu \mathrm{m}$ diamond suspension (MetaDi Supreme) on a MicroCloth and $1 \mu \mathrm{m} \mathrm{Al}_{2} \mathrm{O}_{3}$ (MicroPolish II) on a Microcloth with a polishing machine (MetaServ 250/vector head), all purchased at Bühler $\mathrm{GmbH}$ (Germany). Each polishing step was performed for at least 5 minutes and the crystal was sonicated at least five times in ultrapure water before moving on to the next step, mitigating Pt contamination on the Sn crystals.

Electrochemical deposition procedure. Potential-controlled electrochemical deposition $(-0.10$, $\left.-0.15,-0.25 \mathrm{~V}_{\mathrm{RHE}}\right)$ was carried out by introducing $\mathrm{K}_{2} \mathrm{PtCl}_{4}(99.999 \%$, Sigma Aldrich Corp., Germany) to the Ar-saturated electrolyte while the electrode was rotated at $200 \mathrm{rpm}$. The Pt precursor was dissolved in borate buffer $(1 \mathrm{~mL})$ prior to addition and the amount of $\mathrm{K}_{2} \mathrm{PtCl}_{4}$, added to the cell, was adjusted to yield an overall concentration of $1.5 \mathrm{mM}$. After the desired time of deposition was reached, the electrode was immediately removed from the solution and rinsed with ultrapure water to remove any remaining Pt ions from the surface of the electrode.

Pt sputtering and scanning electron microscopy (SEM). Platinum was sputtered on polished Sn samples in Ar atmosphere (0.05 mbar), using a sputtering machine (Sputter Coater SCD 004, Fluke Corp., USA) with a working distance of $5 \mathrm{~cm}$ at an applied current of 15,45,60, 30 or $60 \mathrm{~A}$ for 40, 30, 26, 180 or $52 \mathrm{~s}$ to achieve a Pt overlayer thickness of 5, 20, 40, 60 or $80 \mathrm{~nm}$. Images of the deposits were taken with a high resolution scanning electron microscope (JCM-7500 F, Jeol Germany GmbH, Germany) after washing the samples with ultrapure water and drying them for at least $24 \mathrm{~h}$ at room temperature. EDX spectroscopy was carried out on a table top SEM-EDX device (JCM-6000, Jeol Germany GmbH, Germany) at $15 \mathrm{kV}$.

XPS characterization. The XPS measurements were performed in a Kratos Axis Supra spectrometer with a monochromatic $\mathrm{Al} \mathrm{K \alpha}$ X-ray source. The PtSn samples were mounted non-conductive in the sample holder by using a carbon tape and a PTFE sheet to adjust the sample's height. The binding energies for each spectra were corrected using the $\mathrm{C} 1 \mathrm{~s}$ peak $(284.6 \mathrm{eV})$ as a reference. The peak areas from the Pt $4 \mathrm{f}$ and $\mathrm{Sn} 3 \mathrm{~d}$ level were calculated with casaXPS processing software. A Shirley background was employed for the background subtraction during the area calculation.

\section{Results and Discussion}

Electrochemistry of Sn. As a non-noble metal, Sn readily forms an oxide on its surface if exposed to an oxidizing agent, such as $\mathrm{O}_{2}$ in air. Furthermore, $\mathrm{Sn}$ dissolves to $\mathrm{Sn}(\mathrm{II})$ and $\mathrm{Sn}$ (IV) species in acidic and alkaline media $^{14}$. In contrast to this, metallic $\mathrm{Sn}$ is thermodynamically stable between approximately -0.1 and $-1.0 \mathrm{~V}_{\mathrm{RHE}}$ in solutions with neutral $\mathrm{pH}^{15}$. Therefore, $\mathrm{Sn}$ can be reversibly oxidized and reduced by potential control in neutral media, e.g., without significant dissolution into the electrolyte ${ }^{16}$. Accordingly, the steady-state CV of a Sn electrode in a borate buffer solution with a $\mathrm{pH}$ of 8.4 is presented in Fig. 1, showing oxidative features at potentials more anodic than $-0.1 \mathrm{~V}_{\mathrm{RHE}}$, as well as reductive features at more cathodic potentials. The electrochemical behavior of $\mathrm{Sn}$ has been studied in various electrolytes by different groups ${ }^{16-19}$, and the following reaction scheme was proposed for the electrochemical oxidation of metallic $\mathrm{Sn}$ in borate buffer by Kapusta et al. ${ }^{16}$.

$$
\mathrm{Sn}+2 \mathrm{OH}^{-} \rightarrow \mathrm{Sn}(\mathrm{OH})_{2}+2 \mathrm{e}^{-}
$$




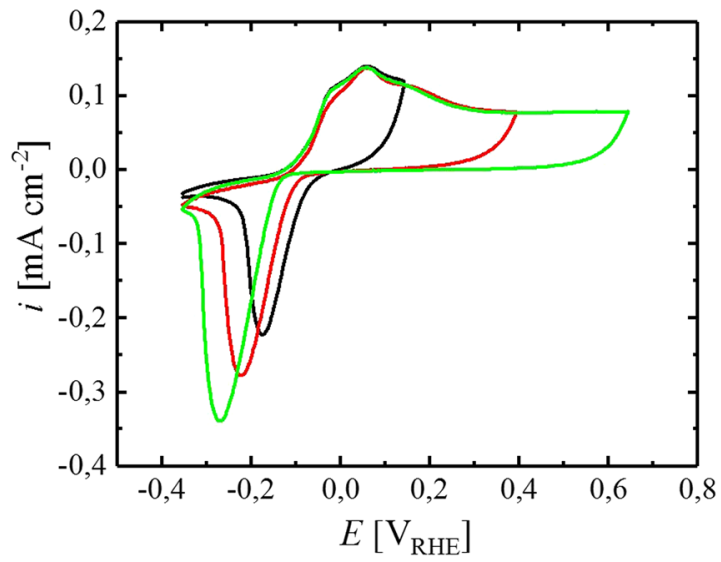

Figure 1. CVs of a Sn electrode in Ar-saturated borate buffer $(0.05 \mathrm{M}, \mathrm{pH}=8.4)$ at a scan rate of $20 \mathrm{mV} \mathrm{s}^{-1}$ between -0.35 and 0.14 (black), 0.39 (red) or $0.64 \mathrm{~V}_{\mathrm{RHE}}$ (green) without rotation, measured at room temperature. Origin 9.1 (https://www.originlab.com).

$$
\mathrm{Sn}(\mathrm{OH})_{2}+2 \mathrm{OH}^{-} \rightarrow \mathrm{Sn}(\mathrm{OH})_{4}+2 \mathrm{e}^{-}
$$

In accordance with these reports, the anodic current shown in Fig. 1 is assigned to the oxidation of the $\mathrm{Sn}$ surface, whereas the cathodic peak is ascribed to the associated reduction to metallic $\mathrm{Sn}$. In the absence of a continuous reaction (e.g., HER), the extent of reversibility of the redox process can be estimated by dividing the integral of the reductive charge $\left(C_{\text {red }}\right)$ by the oxidative charge $\left(C_{\mathrm{ox}}\right)$, passing through the electrode during a single cycle. Considering the $\mathrm{CV}$ with an anodic potential limited of $0.14 \mathrm{~V}_{\mathrm{RHE}}$ (Fig. 1, black line), the oxidative and cathodic charges are perfectly balanced $\left(C_{\mathrm{red}} / C_{\mathrm{ox}} \approx 1\right)$, hence the process of surface oxide formation on $\mathrm{Sn}$ can be considered fully reversible within this potential range. When the anodic vertex of the $\mathrm{CV}$ is increased to higher potentials ( 0.39 and $\left.0.64 \mathrm{~V}_{\mathrm{RHE}}\right)$, the oxidative as well as the reductive charge increases and the cathodic peak shifts to more negative potentials (Fig. 1, red and green lines). This observation can be explained by the transformation of the anodically formed oxide to a more stable oxide layer on the Sn surface at these higher anodic potentials, leading to an increase in the reduction overpotential in the negative going $\operatorname{scan}^{16}$. Furthermore, the charge balance $C_{\text {red }} / C_{\text {ox }}$ decreases to $\approx 0.7$ and $\approx 0.6$ when the anodic vertex increases to 0.39 and $0.64 \mathrm{~V}_{\mathrm{RHE}}$, respectively. The higher oxidative compared to reductive charge indicates an incomplete reduction of the oxide layer within the applied potential region, most likely due to the anodic stabilization of the surface oxide. In alignment with this hypothesis, Kaputsa et al. reported that full removal of certain, anodically grown Sn oxide species requires extended periods at substantially more cathodic potentials, including the formation of $\mathrm{H}_{2}$ on the electrode ${ }^{16}$.

The role of surface oxides in the electrodeposition of $\mathrm{Pt}$. In general, the deposition of metals on oxides is associated with a large interfacial energy, often leading to the formation of islands due to a dewetting effect ${ }^{20}$. The effect of $\mathrm{Sn}$ oxide on the electrochemical deposition of $\mathrm{Pt}$ was studied here using CV in borate buffer solution. Pt deposition on Sn was carried out after potential cycling of the electrode surface in the potential range of the fully reversible oxide formation (i.e., between -0.35 and $0.14 \mathrm{~V}_{\mathrm{RHE}}$ ) until a stable voltammogram was established, whereas high anodic potentials were avoided to abstain from the irreversible oxide formation. When the $\mathrm{CV}$ of the $\mathrm{Sn}$ electrode reached the anodic vertex of $0.14 \mathrm{~V}_{\mathrm{RHE}}$, an already dissolved $\mathrm{Pt}(\mathrm{II})$ precursor $\left(\mathrm{K}_{2} \mathrm{PtCl}_{4}\right)$ was added to the electrolyte and the potential was scanned in cathodic direction. Hence, an oxide is present on the surface of the electrode when the precursor is introduced to the system. Figure 2a shows the CV prior to addition of the Pt precursor (black line) and the subsequent cathodic scan in the presence of Pt ions (red line). With respect to thermodynamics, facile Pt deposition on the electrode surface is expected in the entire applied potential region, due to the large cathodic overpotential of more than $1 \mathrm{~V}$ with respect to the standard reduction potential of $\mathrm{PtCl}_{4}{ }^{2-}\left(0.755 \mathrm{~V}_{\mathrm{SHE}}\right)$ according to the following reaction scheme ${ }^{21}$.

$$
\mathrm{PtCl}_{4}^{2-}+2 \mathrm{e}^{-} \rightarrow \mathrm{Pt}+4 \mathrm{Cl}^{-}
$$

Nevertheless, as shown (red line) in Fig. 2a, the current in the cathodic sweep after the addition of the Pt precursor initially follows the previous cycle, where no $\mathrm{PtCl}_{4}{ }^{2-}$ ions were present in the electrolyte. The absence of a cathodic current in addition to that originating from the redox process on the Sn electrode indicates that Pt deposition from a borate buffer solution does not occur on the $\mathrm{Sn}$ electrode between 0.14 and $\approx-0.12 \mathrm{~V}_{\mathrm{RHE}}$. Nevertheless, the current increases significantly when the potential is scanned further cathodic into the range corresponding to the reduction of Sn oxide species, indicating the onset of Pt deposition. Sweeping the potential further cathodic leads to a linear increase of the current, accounting for ongoing reduction of Pt ions from solution and HER on the resulting deposits. According to these findings, we hypothesize that Pt deposition on Sn is not possible under the applied conditions as long as the metallic surface is covered with oxide species. It can be excluded that the observed phenomenon is due to a time transient effect, where the Pt precursor would not be present at the surface of the electrode until the increase of the current is observed, since the electrode was rotated $(400 \mathrm{rpm})$ during the experiment, ensuring a quick transport of the already dissolved precursor to the electrode 


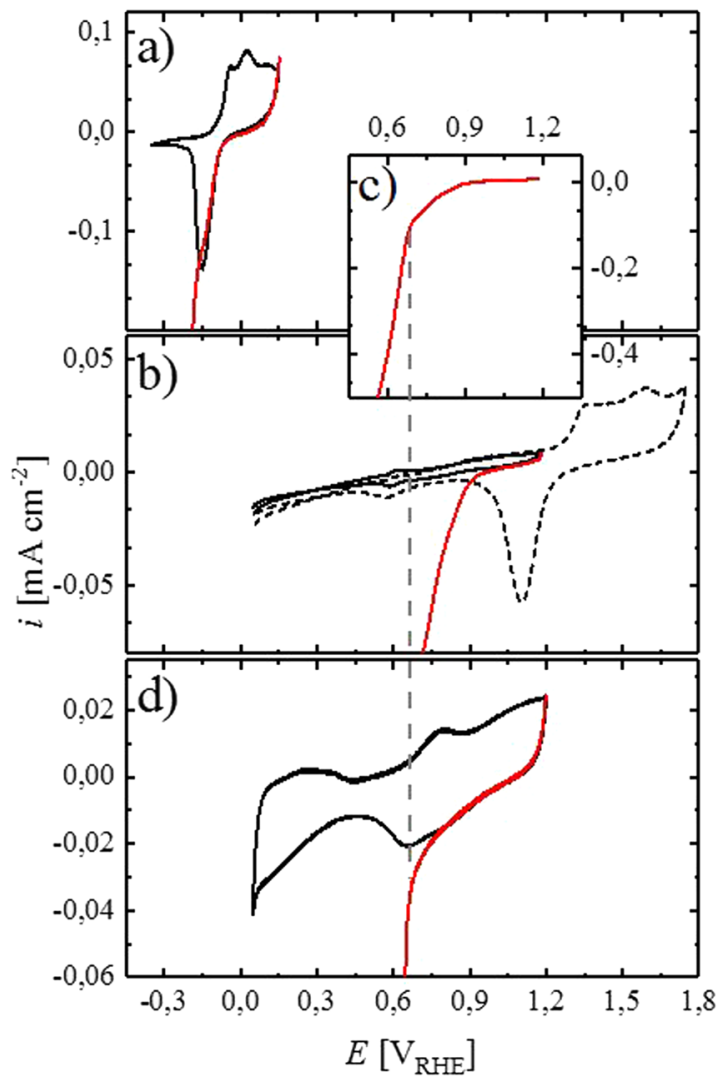

Figure 2. The solid, black lines show CVs of a) $\mathrm{Sn}\left(-0.35-0.14 \mathrm{~V}_{\mathrm{RHE}}\right)$, b) $\mathrm{Au}\left(0.05-1.18 \mathrm{~V}_{\mathrm{RHE}}\right)$ with a zoomed inset in c) and d) Pt (0.05-1.20 $\left.\mathrm{V}_{\mathrm{RHE}}\right)$ in Ar-saturated borate buffer $(0.05 \mathrm{M}, \mathrm{pH}=8.4)$ at a scan rate of $10 \mathrm{mV} \mathrm{s}^{-1}$, applying a rotation rate of $400 \mathrm{rpm}$, measured at room temperature. At the anodic vertex of the CV, a solution containing $\mathrm{K}_{2} \mathrm{PtCl}_{4}(1.5 \mathrm{mM})$ was added to the electrolyte, with the red lines showing the cathodic scan directly after addition of the Pt precursor. The dotted, black line in b) shows a CV of a Au electrode at more anodic potentials (0.05-1.75 $\left.\mathrm{V}_{\mathrm{RHE}}\right)$, as a comparison. The dashed, grey line is solely a guide for the eye to indicate the change of slope in the deposition transient. Origin 9.1 (https://www.originlab.com).

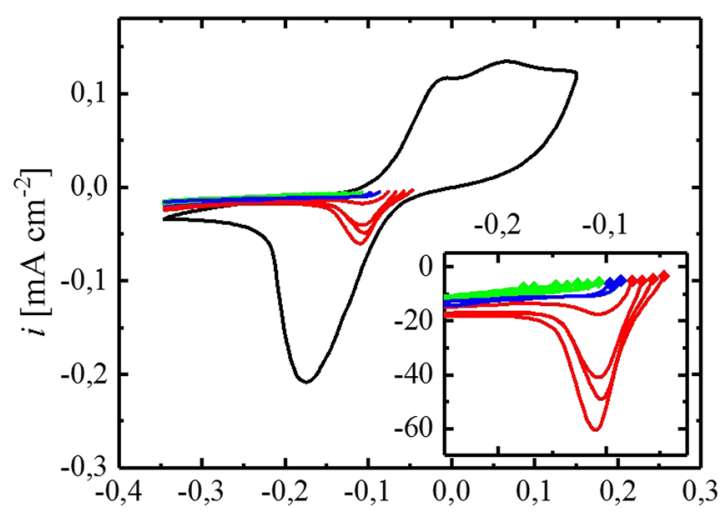

Figure 3. Steady state CV (black line) of a Sn electrode in Ar-saturated borate buffer $(0.05 \mathrm{M}, \mathrm{pH}=8.4)$ at a scan rate of $20 \mathrm{mV} \mathrm{s}^{-1}$ between -0.35 and $0.14 \mathrm{~V}_{\mathrm{RHE}}$, measured at room temperature. Red, blue and green lines show linear potential scans in cathodic direction at $20 \mathrm{mV} \mathrm{s}^{-1}$ after a constant potential was applied for $120 \mathrm{~s}$. The applied potential is indicated by a diamond, which can be seen better in the inset, which shows a zoom of the same graph. Origin 9.1 (https://www.originlab.com).

surface. Furthermore, similar experiments were performed at different potentials (shown at a later point of the study, Fig. 4) where the insertion of the precursor led to an instant rise of the cathodic current. In order to verify that surface oxides hinder the electrochemical deposition process on $\mathrm{Sn}$, a similar experiment was carried out on a Au electrode. Compared to Sn, the electrochemical oxidation of Au in borate buffer takes place at substantially 


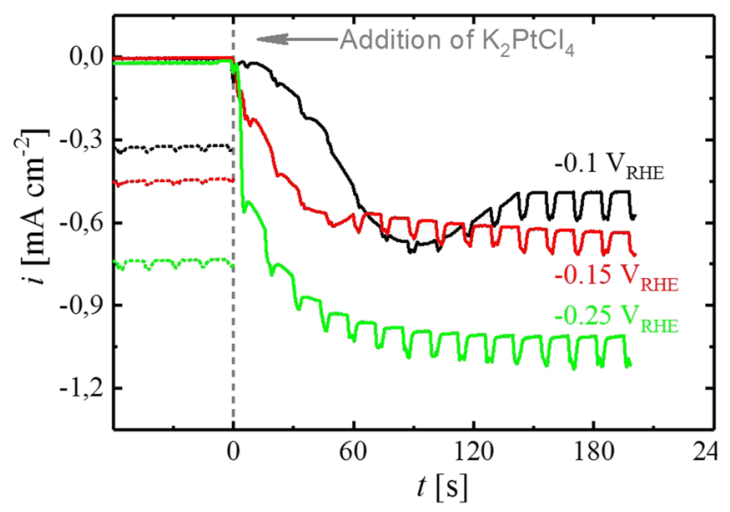

Figure 4. Current transient of potentiostatic measurements on Sn electrodes at -0.1 (black), -0.15 (red) and $-0.25 \mathrm{~V}_{\mathrm{RHE}}$ (green) in deaerated borate buffer $(0.05 \mathrm{M}, \mathrm{pH}=8.4)$ at a rotation rate of $200 \mathrm{rpm}$, interrupted by a $3 \mathrm{~s}$ rotational pulse of $3000 \mathrm{rpm}$ every $15 \mathrm{~s}$, measured at room temperature. A borate buffer solution, containing $\mathrm{K}_{2} \mathrm{PtCl}_{4}$, was added to the electrolyte (time of addition depicted by a dotted, grey line), resulting in an overall concentration of $1.5 \mathrm{mM}$. Origin 9.1 (https://www.originlab.com).

more anodic potentials $>1.2 \mathrm{~V}_{\mathrm{RHE}}$ (dashed, black line, Fig. 2b). Limiting the anodic vertex of the CV to a potential before the oxidation of $\mathrm{Au}$ ensures the metallic state of the electrode, hence no reductive peaks appear in the cathodic scan of the CV (solid, black line, Fig. 2b). By adding the Pt precursor at $1.2 \mathrm{~V}_{\mathrm{RHE}}$ and scanning the potential cathodic, the onset of Pt deposition on Au was found at $\approx 0.9 \mathrm{~V}_{\mathrm{RHE}}$ (red line, Fig. $2 \mathrm{~b}$ ). Owing to the fact that this potential lies more than $1 \mathrm{~V}$ anodic of that found on Sn, we conclude that Pt deposition on Sn is indeed fully prevented in the presence of an oxide layer on the surface. Continuing the cathodic sweep reveals a change of slope with respect to the increase in the deposition current at a potential of approximately $0.66 \mathrm{~V}_{\mathrm{RHE}}$, best visible in Fig. 2c. The charge that passed the electrode in the cathodic scan before reaching $0.66 \mathrm{~V}_{\mathrm{RHE}}$, is approximately $720 \mu \mathrm{C} \mathrm{cm}{ }^{-2}$. Considering a $2 \mathrm{e}^{-}$process for the reduction of $\mathrm{PtCl}_{4}{ }^{2-}$ according to Equation [3], and assuming $100 \%$ efficiency, this charge corresponds approximately to the deposition of one Pt monolayer on Au (assuming a hypothetical roughness factor of $\approx 1.5 \mathrm{~cm}_{\mathrm{pt}}^{2} \mathrm{~cm}^{-2}$ for the Au disk). Hence, the majority of the Au surface is already covered with Pt when the potential of $0.66 \mathrm{~V}_{\mathrm{RHE}}$ is reached. Repeating the deposition experiment on a Pt electrode (Fig. 2d) gives further insight into the Pt deposition process. Similar to Sn, deposition of Pt on Pt starts after the removal of surface oxides on the metal (red line, Fig. 2d). The onset of Pt deposition on the Pt disk was $\approx 0.66$ $\mathrm{V}_{\mathrm{RHE}}$, coinciding well with the potential where a change of slope was observed when Pt is deposited on the Au disk (red line, Fig. 2b). Hence, we hypothesize that Pt deposited on Au at potentials more anodic than $0.66 \mathrm{~V}_{\mathrm{RHE}}$ is oxidized quickly, forming a layer of oxide species. This layer may hinder the continuation of the deposition process, similar to that on a $\mathrm{Pt}$ (or $\mathrm{Sn}$ ) electrode and might eventually stop the process entirely in a certain potential region. By sweeping the potential further cathodic, metallic Pt is exposed and Pt deposition can continue unabated, leading to an increase in the deposition current, thus a change of slope in the cathodic scan of Fig. $2 \mathrm{~b}$ is observed. According to the results presented here, we propose that oxide species on the surface of Pt and Sn electrodes are capable of fully inhibiting Pt deposition under the conditions applied here, even though high cathodic overpotentials are applied to the system. As mentioned earlier, similar self-limiting mechanisms with respect to the deposition of Pt were observed by other researchers who utilized this effect to prepare Pt-ML structures on Au. Brimaud et al. deposited Pt electrochemically on $\mathrm{Au}$ in the presence of CO in the electrolyte. They reported that the rapid adsorption of carbon monoxide on the Pt surface fully blocks further electrodeposition ${ }^{10}$. Liu et al. employed a similar method to obtain a Pt-ML on $\mathrm{Au}$, where they used the underpotential deposition of hydrogen $\left(\mathrm{H}_{\text {upd }}\right)$ to stop the Pt deposition after the formation of the first layer ${ }^{11}$. If developed further, the mechanism reported in this publication could potentially be utilized in a similar way to obtain a Pt-ML or thin film on a non-noble metal support.

Since Pt deposits only on metallic Sn, an experiment was designed to identify the potential at which Sn oxide formation takes place on the surface of the electrode. First of all, a CV, terminating at a potential of $-0.35 \mathrm{~V}_{\mathrm{RHE}}$, was carried out to reduce the surface oxide of the Sn electrode. Thereafter, the potential of interest was applied for 2 minutes (chronoamperometric period) to either stabilize the surface in the metallic state or to form surface oxide species. Subsequently, the potential was scanned cathodic to probe for the appearance of a peak, corresponding to the reduction of the surface oxide to metallic $\mathrm{Sn}$. When potentials more negative than $-0.1 \mathrm{~V}_{\mathrm{RHE}}$ are applied during the chronoamperometric period, no peak appears in the cathodic scan (green lines, Fig. 3), thus no oxidation of the metallic surface has taken place and the probed potential is considered suitable for the electrodeposition of Pt. In contrast to that, clear reduction features are observed after applying potentials more positive than $-0.09 \mathrm{~V}_{\mathrm{RHE}}$ (red lines, Fig. 3) due to (partial) oxidation of the Sn surface. In the transition region between those two potential regions, no clear peak is found but slight reoxidation of the surface cannot be fully excluded (blue lines, Fig. 3). 
The nature of electrodeposited Pt on Sn. In order to obtain an insight into the deposition process, as well as into the morphology of the resulting Pt deposits, potentiostatic electrodeposition was carried out at different potentials and the deposits were imaged by SEM. Furthermore, the change in the electrochemical properties of the Pt deposits, as well as the increase in the film thickness throughout the deposition process with respect to the deposition time are addressed in this section.

To ensure the metallic state of Sn prior to the electrodeposition process, potentiostatic Pt deposition was solely carried out at sufficiently cathodic potentials, ensuring the absence of oxide species on Sn according to Fig. 4, viz., $-0.1,-0.15$ and $-0.25 \mathrm{~V}_{\mathrm{RHE}}$. Prior to deposition, the $\mathrm{Sn}$ oxide on the electrode surface was reduced by scanning the potential cathodic to $-0.35 \mathrm{~V}_{\mathrm{RHE}}$, followed by a stabilization period at the respective deposition potential for $120 \mathrm{~s}$ (in the absence of Pt ions in the solution). Thereafter, $\mathrm{K}_{2} \mathrm{PtCl}_{4}$ pre-dissolved in borate buffer was added to the solution to initiate Pt deposition on Sn. During the electrochemical deposition process, a rotation rate of $200 \mathrm{rpm}$ was applied, interrupted every $15 \mathrm{~s}$ by a rotational pulse of $3000 \mathrm{rpm}$ for $3 \mathrm{~s}$ to remove hydrogen bubbles from the surface of the electrode.

As shown in Fig. 4 (solid lines), the current at each potential remains constant before the addition of the Pt precursor, indicating steady conditions and the absence of additional reductive processes, e.g., due to the removal of residual surface oxides. As soon as $\mathrm{K}_{2} \mathrm{PtCl}_{4}$ is introduced to the electrolyte, the current increases in all experiments, eventually sloping out to a plateau for the remainder of the potentiostatic period. Therefore, the current increase can clearly be ascribed to the reduction of Pt ions at the surface of the electrode, superimposed with a co-current from HER on the Pt deposits. In contrast to $\mathrm{Sn}^{22}$, Pt is highly active for HOR/HER in a wide range of $\mathrm{pH}$ values ${ }^{23-27}$. Therefore, a significant increase of the current is expected for a Sn electrode once it is at least partially covered with Pt due to the large applied HER overpotential. Consistently, the cathodic current after the addition of the Pt precursor originates from Pt deposition, as well as from HER on the Pt deposits. The application of a larger HER overpotential (more cathodic potential) therefore causes a higher current during the plateau phase. Moreover, the time needed to reach the plateau is clearly dependent on the applied potential, with more cathodic potentials enhancing the rate of the deposition process. As a result, the Sn surface is more quickly covered with Pt at more cathodic potentials and the plateau, corresponding to the maximum rate of HER, is reached more quickly. Consistently, the current measured at $-0.1 \mathrm{~V}_{\mathrm{RHE}}$ (Fig. 4, black line) requires significantly more time to reach steady state and reproducibly shows a current peak before the final plateau. We account this to an overlap of Pt deposition on uncovered Sn sites with the current originating from HER on Pt, since covering the electrode with Pt requires more time compared to -0.15 (Fig. 4, red line) and $-0.25 \mathrm{~V}_{\mathrm{RHE}}$ (Fig. 4, green line). However, according to Strmcnik et al., the surface of $\mathrm{Pt}$ is fully covered with $\mathrm{H}_{\mathrm{ads}}$ in the relevant potential region $(\leq-0.1$ $\left.\mathrm{V}_{\mathrm{RHE}}\right)^{28}$. Liu et al. utilized the rapid formation of $\mathrm{Pt}-\mathrm{H}_{\mathrm{ads}}$ on the surface of the deposited Pt to prepare a Pt-ML on a single crystalline gold surface, where Pt electrodeposition was fully prevented by the adsorbed hydrogen ${ }^{11}$. Since the potential range utilized for deposition experiments in this study is similar to that of Liu et al. $(-0.33$ $\mathrm{V}_{\mathrm{RHE}}$ ), one might expect a comparable inhibition of the deposition process on Sn. However, the electrolyte used by Liu et al. was an unbuffered $\mathrm{NaCl}$ solution $(0.5 \mathrm{M})$, where the $\mathrm{pH}$ at the surface of the electrode shifts due to the consumption of $\mathrm{H}^{+}$during HER on $\mathrm{Pt}^{12}$. The advantage of this approach is the possibility to adjust the rate of $\mathrm{H}_{2}$ evolution to a moderate level by choosing the solution $\mathrm{pH}$ accordingly. Hence, using an electrolyte with a $\mathrm{pH}$ of 4 (according to Liu et al.) results in an HER limiting current of $\approx 100 \mu \mathrm{A} \mathrm{cm}^{-2}$ at a rotation rate of $400 \mathrm{rpm}^{11}$. In this case, the associated molar flux of $\mathrm{H}_{2}$ produced at the surface of the electrode is as low as $\approx 0.5 \mathrm{nmol} \mathrm{s} \mathrm{cm}^{-2}$ (calculated via Faraday's law at a current density of $100 \mu \mathrm{A} \mathrm{cm}^{-2}$ in a $2 \mathrm{e}^{-}$transfer reaction). In contrast to this rather low $\mathrm{H}_{2}$ production rate, the maximum flux transported away from the electrode through diffusion under the applied conditions is on the order of $15 \mathrm{nmol} \mathrm{s} \mathrm{cm}^{-2}$ (calculated via Fick's first law, assuming a diffusion boundary layer thickness of $40 \mu \mathrm{m}$ at $400 \mathrm{rpm}$, a maximum $\mathrm{H}_{2}$ concentration of $1.3 \mathrm{mM}$ at $25^{\circ} \mathrm{C}^{25}$, a diffusion coefficient of $4.5 \cdot 10^{-9} \mathrm{~m}^{2} \mathrm{~s}^{-1}$ and the absence of $\mathrm{H}_{2}$ in the bulk of the solution) ${ }^{29}$, allowing the produced $\mathrm{H}_{2}$ to be easily transported away from the electrode into the bulk of solution without forming bubbles on the electrode surface. In accordance with this, a solution $\mathrm{pH}$ of 8.4, i.e., as used in all $\mathrm{Pt}$ deposition experiments in this article, results in a theoretical limiting current of $12 \mathrm{nA} \mathrm{cm}^{-2}$ (calculated via the Levich equation using a diffusion coefficient of $7 \cdot 10^{-9} \mathrm{~m}^{2} \mathrm{~s}^{-1}$ and a rotation rate of $\left.400 \mathrm{rpm}\right)^{30}$ due to the comparably low proton concentration. However, in unbuffered electrolytes, the concentration of protons at the surface of the electrode depletes when HER proceeds, resulting in a shift of the surface $\mathrm{pH}$. Hence, due to the limited stability window of Sn with respect to $\mathrm{pH}$ (Sn dissolves in acidic and alkaline media) $)^{15}$, a buffered electrolyte was used in all experiments described here. Since protons can be readily replenished in buffers, a change of the solution $\mathrm{pH}$ is avoided and the $\mathrm{H}^{+}$concentration in the vicinity of the electrode does not deplete analogously to an unbuffered electrolyte. Therefore, the current observed on a polycrystalline Pt disk (Fig. 4, dotted lines) in the same electrolyte, at the potentials used for electrochemical deposition, i.e., $-0.10,-0.15$ and $-0.25 \mathrm{~V}_{\mathrm{RHE}}$, ranges between $0.34-0.76 \mathrm{~mA} \mathrm{~cm}^{-2}$ at a rotation rate of $400 \mathrm{rpm}$. Hence, the rate of $\mathrm{H}_{2}$ evolution at the surface of the electrode is approximately one order of magnitude larger compared to Liu et al. Accordingly, $\mathrm{H}_{2}$ bubbles can form on the surface of the electrode, eventually being released into the electrolyte. The surface sites liberated in this process may serve as template for further Pt deposition, hence the deposition process is not expected to be fully inhibited under the conditions applied here. Nevertheless, it is expected that the rate of Pt deposition is significantly decreased due to blockage of $\mathrm{Pt}$ sites by adsorbed hydrogen. In principle, a rough estimation of the efficiency of the deposition process during the plateau phase can be made by subtraction of the charge originating from HER on pure Pt from the charge obtained during the electrodeposition experiments. Dividing this "HER-corrected" charge by the total charge delivered during the same period of time yields a maximum deposition efficiency of 30,23 and $24 \%$ at -0.10 , -0.15 and $-0.25 \mathrm{~V}_{\mathrm{RHE}}$. Even though an extraction of the amount of deposited Pt by this method is rather delicate due to differences in the nature of the electrode (Pt deposits on Sn versus pure $\mathrm{Pt}$ ), the electrode morphology, and the additional salt in the electrolyte, the low calculated efficiency indicates that the deposition of Pt on the electrodes is strongly limited. 


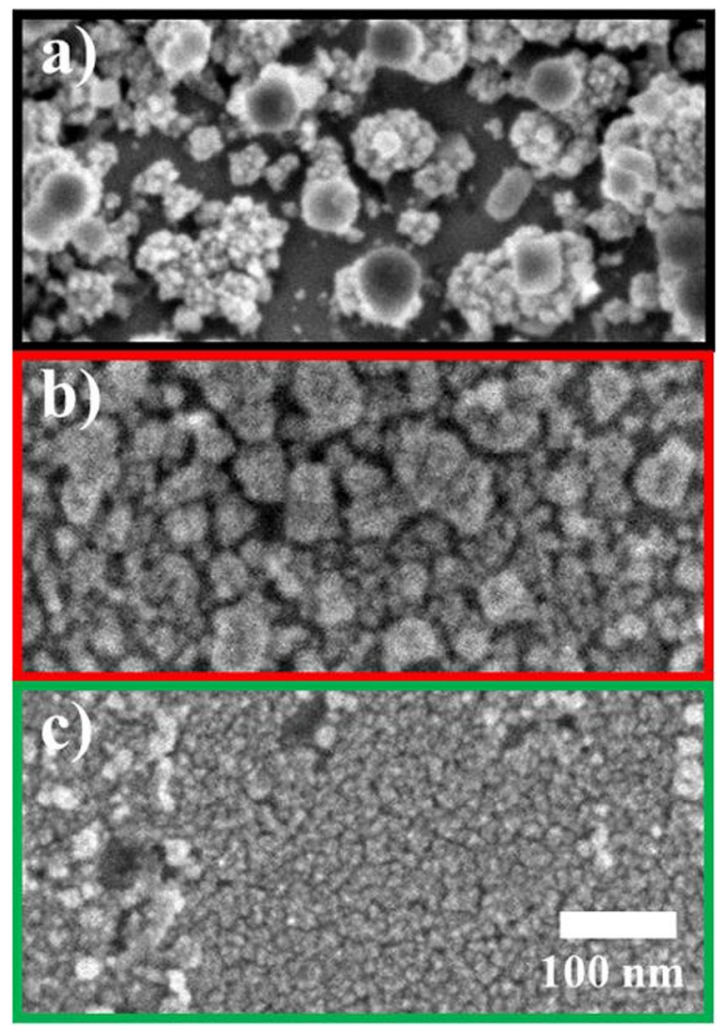

Figure 5. SEM images of $\mathrm{Pt}$ deposits on $\mathrm{Sn}$, prepared by electrodeposition in borate buffer $(\mathrm{pH}=8.4,0.05 \mathrm{M})$ at (a) $-0.10,(\mathbf{b})-0.15$ and (c) $-0.25 \mathrm{~V}_{\mathrm{RHE}}$ for $204 \mathrm{~s}$, applying a rotation rate of $200 \mathrm{rpm}$, interrupted by a $3 \mathrm{~s}$ rotational pulse of $3000 \mathrm{rpm}$ every $15 \mathrm{~s}$.

This is then also reflected in the morphology of the resulting deposits, which is shown in the SEM images of the electrodes after the electrodeposition experiment (Fig. $5 \mathrm{a}-\mathrm{c}$ ). In general, the electrodes are composed of a variety of small, well-connected Pt deposits with an island-like morphology. The lateral size of the deposits varies with respect to the applied deposition potential in the order $-0.1>-0.15>-0.25 \mathrm{~V}_{\mathrm{RHE}}$. The average island diameter at the most cathodic deposition potential of $-0.25 \mathrm{~V}_{\mathrm{RHE}}$ (Fig. $5 \mathrm{c}$ ) is on the order of $10 \mathrm{~nm}$, while deposits produced at -0.15 and $-0.1 \mathrm{~V}_{\mathrm{RHE}}$ have slightly larger dimensions. We conclude that the higher overpotential with respect to the HER on Pt at more cathodic potentials, causes the evolution of large amounts of $\mathrm{H}_{2}$ on the surface of the Pt deposits. This ongoing reaction shields the Pt deposits from successive reduction of ions on their surface, while pure Sn sites are more exposed to the solution. Hence, Pt deposition on Sn is favored over the deposition on the Pt islands and smaller deposits are formed. This, however, implies that the average film thickness would not grow significantly during longer potential application, since most of the charge in the plateau phase would correspond to HER as soon as a high coverage on the electrode is achieved. It shall be mentioned at this point, that the effect of the high $\mathrm{H}_{2}$ concentration in the vicinity of the electrode on the chemical stability of the $\mathrm{PtCl}_{4}{ }^{2-}$ complex was not studied here and a certain amount of chemical deposition due to reduction of $\mathrm{Pt}$ ions by $\mathrm{H}_{2}$ cannot be fully excluded.

To further investigate the Pt deposition efficiency during the plateau phase, electrochemical deposition using the same procedure employed earlier was carried out at a constant potential of $-0.25 \mathrm{~V}_{\mathrm{RHE}}$, varying the time from 68,190 to $626 \mathrm{~s}$ (termed 1, 3 and 10 min hereafter). While the shortest deposition time is still in the region where the current increases, the other two deposition times were chosen such that the current plateau was reached, remaining at these conditions for a different amount of time. The equivalent thickness of the resulting deposits was analyzed by EDX, where the electron beam penetrates through the Pt surface layer into the Sn metal. Therefore, the ratio of the X-rays emitted by $\mathrm{Pt}$ and $\mathrm{Sn}$ contains intrinsic information about the thickness of the Pt overlayer. The Pt layer thickness was estimated by comparing the Pt:Sn count ratio to those obtained from samples of defined Pt layer thickness on Sn, obtained by sputter deposition. The thickness of the sputter-deposited Pt films was controlled using a quartz crystal microbalance (QCMB) in the deposition chamber. As expected, the $\mathrm{Pt}: S n$ ratio of the sputter-deposited samples increases with nominal Pt layer thickness, as shown in Fig. 6 (grey circles). While the increase is nearly linear for thin Pt layers, a strong increase is observed for thicker layers (e.g., $\mathrm{Pt}: \mathrm{Sn} \approx 13$ for $80 \mathrm{~nm}$ ), which we hypothesize to originate from the lower beam penetration through the Pt layer as the thickness increases. Nevertheless, the general correlation of the Pt:Sn count ratio and the Pt overlayer thickness can be used to estimate the thickness of the Pt deposits on Sn, which have a comparably small Pt:Sn ratio of $0.055,0.105$ and 0.114 for 1,3 and 10 min of electrodeposition, shown in the inset of Fig. 6 (turquoise, magenta and purple stars). The obtained values correspond approximately to a thickness of $4 \mathrm{~nm}$ after $1 \mathrm{~min}$ and 7-8 nm after 3 and $10 \mathrm{~min}$. We therefore conclude that the efficiency of Pt deposition is highest in the initial phase, where 


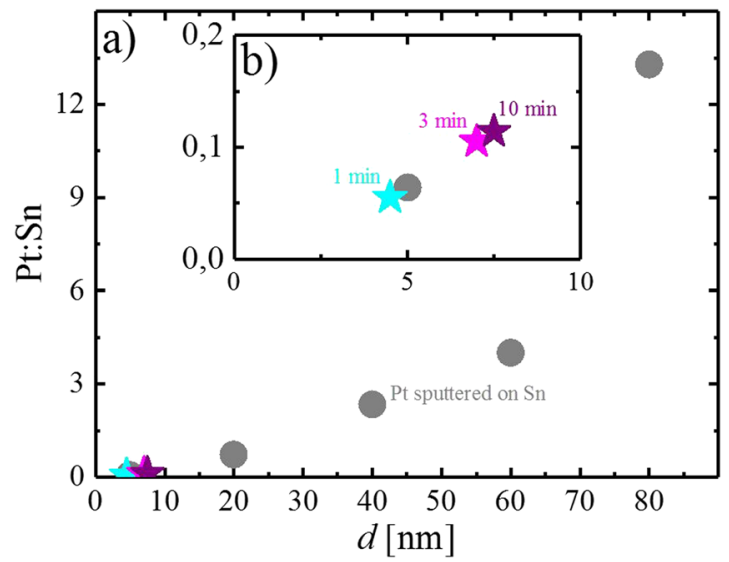

Figure 6. Ratio of the Pt:Sn counts vs. the equivalent thickness, $d$, of the Pt deposit on Sn. The counts were obtained by EDX analysis at $15 \mathrm{kV}$. The Pt layer was either prepared by sputter deposition (grey circles) or by electrochemical deposition for 1 (turquoise star), 3 (magenta star) or $10 \mathrm{~min}$ (purple star). While the full range of prepared samples is shown in a), the inset b) represents a zoom below $10 \mathrm{~nm}$. Origin 9.1 (https://www. originlab.com).

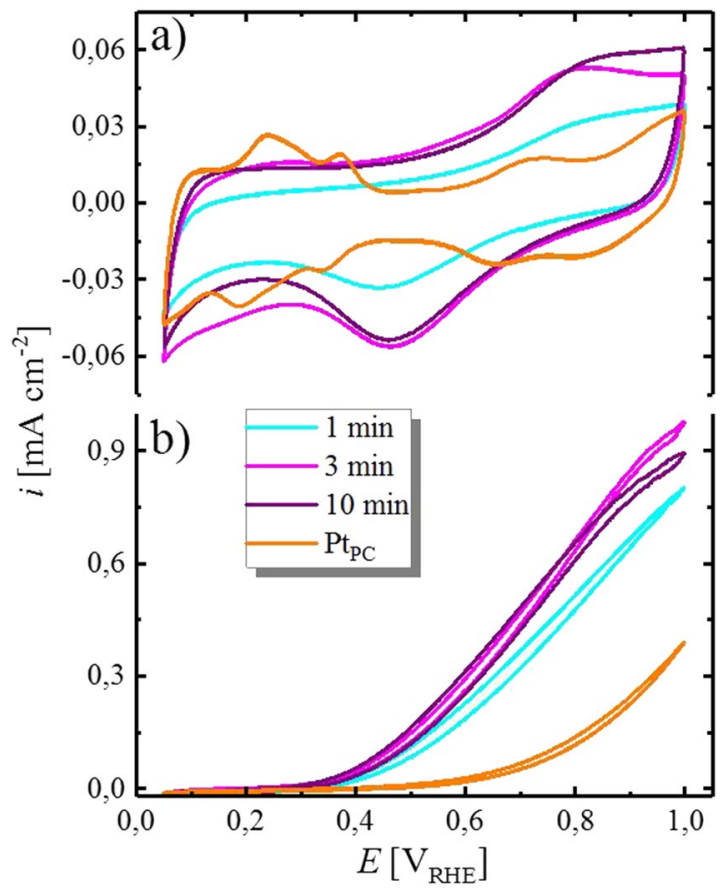

Figure 7. (a) CVs of electrochemically deposited Pt on Sn after a deposition for 1 (turquoise line), 3 (magenta line) and $10 \mathrm{~min}$ (purple line) at $-0.25 \mathrm{~V}_{\mathrm{RHE}}$ in Ar-saturated borate buffer $(0.05 \mathrm{M}, \mathrm{pH}=8.4)$ at a scan rate of $20 \mathrm{mV} \mathrm{s}^{-1}$ between 0.05 and $1.00 \mathrm{~V}_{\mathrm{RHE}}$, measured in stagnant electrolyte at room temperature. The $\mathrm{CV}$ of a polycrystalline $\mathrm{Pt}$ disk is shown as comparison (orange line). (b) CVs of the same deposits after saturating the electrolyte with CO and scanning at $10 \mathrm{mV} \mathrm{s}^{-1}$ and a rotation rate of $400 \mathrm{rpm}$. Origin 9.1 (https://www.originlab.com).

most of the Sn surface sites are available. On the other hand, the deposition process slows down significantly in the plateau phase, where a large fraction of the current originates from HER on the Pt deposits, shielding the electrode surface.

Electrochemical analysis of Pt deposits on Sn. Since the preceding EDX analysis showed that the approximate thickness of the deposits does not increase significantly during the current plateau phase, the analysis of this effect was complemented by measuring CVs of the electrodeposits in borate buffer solution, provided in Fig. 7a. First, it has to be noted that the general shape of the CV for all prepared deposits is similar, whereas the oxidative, as well as the reductive charge of the sample prepared at $1 \mathrm{~min}$ is significantly lower compared to 3 and $10 \mathrm{~min}$. Furthermore, the charge of the $\mathrm{CV}$ of the electrodeposit after $3 \mathrm{~min}$ compares well with that after $10 \mathrm{~min}$ deposition time, indicating a similar exposed surface area. Since the main deposition time for both samples (3 


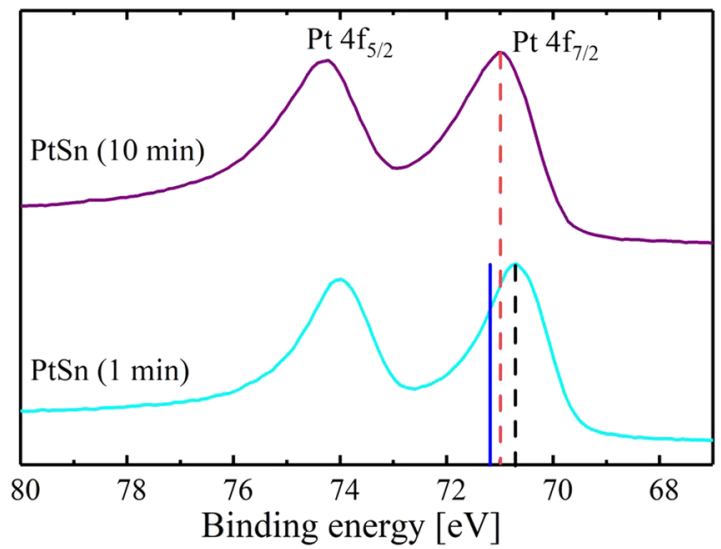

Figure 8. XPS spectra of the Pt $4 \mathrm{f}$ region of PtSn samples after $1 \mathrm{~min}$ (turquoise line) and after $10 \mathrm{~min}$ (purple line) of electrochemical deposition. The blue solid line represents the $\mathrm{Pt} 4 \mathrm{f}_{7 / 2}$ binding energy for a pure $\mathrm{Pt}$ sample reported in the literature ${ }^{35-37}$. Origin 9.1 (https://www.originlab.com) and CasaXPS version 2.3.19PR1.0 (http://www.casaxps.com/).

and $10 \mathrm{~min}$ ) was passed during the plateau phase, the previously stated hypothesis that the deposition during this phase is strongly limited, while the major fraction of the charge originates from HER, is hereby confirmed. Moreover, the charge of the CVs in the oxide formation/removal region $\left(E>0.4 \mathrm{~V}_{\mathrm{RHE}}\right)$ after Pt electrodeposition on $\mathrm{Sn}$ is higher compared to a bare $\mathrm{Pt}_{\mathrm{PC}}$ disk. This is indicative of a high roughness factor $(r f)$ of the $\mathrm{Pt}$ deposit on $\mathrm{Sn}$, stemming from the highly structured surface compared to the flat, polished Pt electrode $(r f \approx 1.3$, extracted from the averaged $\mathrm{H}_{\text {upd }}$ charge in the anodic and cathodic scan). Furthermore, the electrochemical features on the Pt electrodeposits are very different from pure $\mathrm{Pt}_{\mathrm{PC}}$ (orange line), especially at low potentials, where no distinct adsorption features for the $\mathrm{H}_{\text {upd }}$ are present on the electrodeposits. Additionally, the oxidation and reduction potential of the Pt electrodeposit is further separated compared to pure Pt, indicating a larger required overpotential for this process. The differences of the CVs compared to pure Pt can be understood by considering the presence of $\mathrm{Sn}$ in the vicinity of Pt. In fact, Pt-Sn alloys are known to exhibit distinct electrochemical features compared to Pt, visible e.g., in acidic electrolyte ${ }^{31,32}$. This can also be observed in the CVs shown in Fig. $7 \mathrm{a}$, where the oxidative and reductive features of the PtSn catalysts extend over a wider potential range compared to pure $\mathrm{Pt}$ (orange line) and the features in the potential window of the $\mathrm{H}_{\text {upd }}$ are not clearly resolved. Since such alloys are furthermore well-known to be excellent catalysts for the oxidation of carbon monoxide ${ }^{33,34}$, the electrolyte was saturated with CO, reporting steady-state CVs in Fig. $7 \mathrm{~b}$ in order to probe the deposits for a possible alloy formation. Compared to $\mathrm{Pt}_{\mathrm{PC}}\left(\approx 0.6 \mathrm{~V}_{\mathrm{RHE}}\right)$, the onset potential of the oxidation of CO on the Pt deposits on Sn is shifted approximately $200 \mathrm{mV}$ cathodic, which is comparable to the results presented in the literature for PtSn alloys ${ }^{34}$. Additionally, depositing Pt for $1 \mathrm{~min}$ results in a slightly lower activity towards the CO oxidation, which might be mainly related to the incomplete coverage of Pt on the Sn surface, hence a lower $r f$ value, as indicated by the CV in Fig. 7a. Therefore, the formation of a Pt-Sn alloy upon electrochemical deposition from borate buffer solution was confirmed by the CVs and CO oxidation experiments.

To further confirm the formation of the PtSn surface alloy, XPS was used to analyze the Sn samples that were exposed to $1 \mathrm{~min}$ and $10 \mathrm{~min}$ of Pt deposition. Figure 8 shows the spectra of the Pt $4 \mathrm{f}$ level for the two PtSn samples obtained at different deposition times ( $1 \mathrm{~min}$ and $10 \mathrm{~min}$ ). The photoelectron peaks of the PtSn samples show a shift towards lower biding energies when compared to a pure Pt reference obtained from literature ${ }^{35-37}$. Moreover, the Sn ample on which Pt was deposited for 1 min shows the largest shift compared to the Pt reference, with a deviation of $0.5 \mathrm{eV}$. The nature of this shift can be explained by the formation of an alloy between the deposited Pt and Sn on the surface of the electrode ${ }^{35,36}$. On the other hand, the sample on which Pt was deposited for $10 \mathrm{~min}$ shows a smaller shift from the pure Pt reference, suggesting that the alloying between $\mathrm{Pt}$ and $\mathrm{Sn}$ is most likely diluted by pure Pt that couldn't reach the Sn surface.

\section{Conclusions}

Herein, we report insights into the electrochemical deposition process of Pt on Sn from a pH neutral borate buffer solution. Under the tested conditions, Pt electrodeposition was fully prevented when the Sn surface was covered with an oxide layer, even though high cathodic overpotentials were applied. In addition, the same general principle of self-limitation was found to be valid for Pt deposition on a Pt electrode and for a Pt covered Au surface. We conclude from these findings that the concept of surface oxide blocking, introduced here for the first time, has potential to be further developed into a controllable self-limiting deposition process. Such a process could, e.g., be based on the deposition of a Pt-ML on a metallic surface, thereafter oxidizing the surface of the Pt deposit and stopping further deposition.

Furthermore, a method was developed to deposit Pt on Sn in the absence of surface oxide, while HER proceeded on the Pt deposits. In this study, the effect of the applied deposition potential and -time on the resulting morphology were investigated. After an initiation period of a constant potential deposition, where the largest fraction of the Sn surface was covered with Pt, the deposition process was found to be self-limiting due to ongoing HER on Pt. Hence, increasing the deposition time resulted essentially in the same deposited layer thickness 
$(<10 \mathrm{~nm})$ and similar electrochemical behavior of the electrodes. Deposited Pt was found to alloy immediately with the Sn substrate, providing a high activity towards the oxidation of CO. In conclusion, the deposition procedure presented here can be used to reliably obtain Pt overlayers in the nanometer range, alloyed with the substrate in a single step.

Received: 2 September 2019; Accepted: 3 December 2019;

Published online: 09 January 2020

\section{References}

1. Toyota Advanced Technology Communications. Toyota Ushers in the Future with Launch of 'Mirai' Fuel Cell Sedan. Available at, http://newsroom.toyota.co.jp/en/detail/4198334/ (2014).

2. Global, P. R. Team. Hyundai Celebrates World's First Assembly Line Production of Zero-Emissions Fuel Cell Vehicles. Available at, http://globalpr.hyundai.com/prCenter/news/newsView.do?dID=1581 (2013)

3. Honda North America. Southern California Customers Take Delivery of New 2017 Honda Clarity Fuel Cell Sedan. Available at, http://hondanews.com/honda-automobiles/channels/clarity-fuel-cell-press-releases/releases/southern-california-customers-takedelivery-of-new-2017-honda-clarity-fuel-cell-sedan (2016).

4. U.S. DRIVE partnership. Fuel Cell Technical Team Roadmap. Available at, http://energy.gov/sites/prod/files/2014/02/f8/fctt_ roadmap_june2013.pdf (2013).

5. Kinoshita, K. Particle Size Effects for Oxygen Reduction on Highly Dispersed Platinum in Acid Electrolytes. J. Electrochem. Soc. 137, 845, https://doi.org/10.1149/1.2086566 (1990).

6. Reiser, C. A. et al. A Reverse-Current Decay Mechanism for Fuel Cells. Electrochem. Solid-State Lett. 8, A273, https://doi. org/10.1149/1.1896466 (2005).

7. Yu, Y. et al. A review on performance degradation of proton exchange membrane fuel cells during startup and shutdown processes. Causes, consequences, and mitigation strategies. J. Power Sources 205, 10-23, https://doi.org/10.1016/j.jpowsour.2012.01.059 (2012).

8. Mittermeier, T., Weiß, A., Hasché, F., Hübner, G. \& Gasteiger, H. A. PEM Fuel Cell Start-up/Shut-down Losses vs Temperature for Non-Graphitized and Graphitized Cathode Carbon Supports. J. Electrochem. Soc. 164, F127, https://doi.org/10.1149/2.1061702jes (2017).

9. Brankovic, S. R., Wang, J. X. \& Adžić, R. R. Metal monolayer deposition by replacement of metal adlayers on electrode surfaces. Surf. Sci. 474, L173-L179, https://doi.org/10.1016/S0039-6028(00)01103-1 (2001).

10. Brimaud, S. \& Behm, R. J. Electrodeposition of a Pt Monolayer Film: Using Kinetic Limitations for Atomic Layer Epitaxy. J. Am. Chem. Soc. 135, 11716-11719, https://doi.org/10.1021/ja4051795 (2013).

11. Liu, Y., Gokcen, D., Bertocci, U. \& Moffat, T. P. Self-Terminating Growth of Platinum Films by Electrochemical Deposition. Science 338, 1327-1330, https://doi.org/10.1126/science.1228925 (2012).

12. Katsounaros, I. et al. The effective surface $\mathrm{pH}$ during reactions at the solid-liquid interface. Electrochem. Commun. 13, 634-637, https://doi.org/10.1016/j.elecom.2011.03.032 (2011).

13. Liu, Y., Hangarter, C. M., Garcia, D. \& Moffat, T. P. Self-terminating electrodeposition of ultrathin Pt films on Ni: An active, low-cost electrode for H2 production. Surf. Sci. 631, 141-154, https://doi.org/10.1016/j.susc.2014.06.002 (2015).

14. Holleman, A. F., Wiberg, E. \& Wiberg, N. Lehrbuch der anorganischen Chemie. 102nd ed. (de Gruyter, Berlin, New York, 2007).

15. Pourbaix, M. Atlas of electrochemical equilibria in aqueous solutions. 2nd ed. (National Association of Corrosion Engineers, Houston, Texas, 1974).

16. Kapusta, S. D. \& Hackerman, N. Anodic passivation of tin in slightly alkaline solutions. Electrochim. Acta 25, 1625-1639, https://doi. org/10.1016/0013-4686(80)80016-8(1980).

17. El Wakkad, S. E. S., El Din, A. M., Shams \& Sayed, E. l., Jeannette A. The anodic oxidation of metals at very low current density. Part VI. Tin. J. Chem. Soc, 3103, https://doi.org/10.1039/JR9540003103 (1954).

18. Shah, S. N. \& Davies, D. E. The anodic behaviour of tin in alkaline solutions-I. 0.1 M sodium borate solution. Electrochim. Acta 8 , 663-678, https://doi.org/10.1016/0013-4686(63)85012-4 (1963).

19. Palacios-Padrós, A., Caballero-Briones, F., Díez-Pérez, I. \& Sanz, F. Tin passivation in alkaline media. Formation of SnO microcrystals as hydroxyl etching product. Electrochim. Acta 111, 837-845, https://doi.org/10.1016/j.electacta.2013.07.200 (2013).

20. Campbell, C. T. Metal films and particles on oxide surfaces: structural, electronic and chemisorptive properties. Faraday Trans. 92, 1435, https://doi.org/10.1039/FT9969201435 (1996).

21. Harris, D. C. Quantitative chemical analysis. 7th ed. (W. H. Freeman; Palgrave distributor, New York, Basingstoke, 2007).

22. Azizi, O., Jafarian, M., Gobal, F., Heli, H. \& Mahjani, M. G. The investigation of the kinetics and mechanism of hydrogen evolution reaction on tin. Int. J. Hydrogen Energ. 32, 1755-1761, https://doi.org/10.1016/j.ijhydene.2006.08.043 (2007).

23. Neyerlin, K. C., Gu, W., Jorne, J. \& Gasteiger, H. A. Study of the Exchange Current Density for the Hydrogen Oxidation and Evolution Reactions. J. Electrochem. Soc. 154, B631, https://doi.org/10.1149/1.2733987 (2007).

24. Sheng, W., Gasteiger, H. A. \& Shao-Horn, Y. Hydrogen Oxidation and Evolution Reaction Kinetics on Platinum: Acid vs Alkaline Electrolytes. J. Electrochem. Soc. 157, B1529, https://doi.org/10.1149/1.3483106 (2010).

25. Rheinländer, P. J., Herranz, J., Durst, J. \& Gasteiger, H. A. Kinetics of the Hydrogen Oxidation/Evolution Reaction on Polycrystalline Platinum in Alkaline Electrolyte Reaction Order with Respect to Hydrogen Pressure. J. Electrochem. Soc. 161, F1448, https://doi. org/10.1149/2.0501414jes (2014).

26. Durst, J. et al. New insights into the electrochemical hydrogen oxidation and evolution reaction mechanism. Energy Environ. Sci. 7, 2255, https://doi.org/10.1039/c4ee00440j (2014).

27. Sheng, W. et al. Correlating hydrogen oxidation and evolution activity on platinum at different $\mathrm{pH}$ with measured hydrogen binding energy. Nat. Commun. 6, 5848, https://doi.org/10.1038/ncomms6848 (2015).

28. Strmcnik, D., Tripkovic, D., van der Vliet, D., Stamenkovic, V. \& Marković, N. M. Adsorption of hydrogen on Pt(111) and Pt(100) surfaces and its role in the HOR. Electrochem. Commun. 10, 1602-1605, https://doi.org/10.1016/j.elecom.2008.08.019 (2008).

29. Ferrell, R. T. \& Himmelblau, D. M. Diffusion coefficients of hydrogen and helium in water. AIChE J. 13, 702-708, https://doi. org/10.1002/aic.690130421 (1967).

30. Lee, S. H. \& Rasaiah, J. C. Proton transfer and the mobilities of the $\mathrm{H}+$ and $\mathrm{OH}$ - ions from studies of a dissociating model for water. J. Chem. Phys. 135, 124505, https://doi.org/10.1063/1.3632990 (2011).

31. Santos, M. C. \& Bulhões, L. O. S. The underpotential deposition of Sn on Pt in acid media. Cyclic voltammetric and electrochemical quartz crystal microbalance studies. Electrochim. Acta 48, 2607-2614, https://doi.org/10.1016/S0013-4686(03)00304-9 (2003).

32. Colmati, F., Antolini, E. \& Gonzalez, E. R. Pt-Sn/C electrocatalysts for methanol oxidation synthesized by reduction with formic acid. Electrochim. Acta 50, 5496-5503, https://doi.org/10.1016/j.electacta.2005.03.030 (2005).

33. Gasteiger, H. A., Marković, N. M. \& Ross, P. N. Electrooxidation of CO and H2/CO Mixtures on a Well-Characterized Pt3Sn Electrode Surface. J. Phys. Chem. 99, 8945-8949, https://doi.org/10.1021/j100022a002 (1995).

34. Gasteiger, H. A., Marković, N. M. \& Ross, P. N. Structural effects in electrocatalysis: electrooxidation of carbon monoxide on Pt3Sn single-crystal alloy surfaces. Catal. Lett. 36, 1-8, https://doi.org/10.1007/BF00807197 (1996). 
35. Ramallo-López, J. M. et al. XPS and XAFS Pt L 2,3 -Edge Studies of Dispersed Metallic Pt and PtSn Clusters on SiO 2 Obtained by Organometallic Synthesis: Structural and Electronic Characteristics. J. Phys. Chem. B 107, 11441, https://doi.org/10.1021/jp030579y (2003).

36. Siri, G. J. et al. XPS and EXAFS study of supported PtSn catalysts obtained by surface organometallic chemistry on metals. Appl. Catal., A 278, 239, https://doi.org/10.1016/j.apcata.2004.10.004 (2005).

37. Moulder, J. F. \& Chastain, J. Handbook of X-ray Photoelectron Spectroscopy. A Reference Book of Standard Spectra for Identification and Interpretation of XPS Data (Perkin-Elmer Corporation, Eden Prairie, Minn., 1992).

\section{Acknowledgements}

Special thanks to Thomas Mittermeier for fruitful discussions. Furthermore, the authors would like to thank Katia Rodewald for supplying SEM images. Financial support in the frame of the European Community's Seventh Framework Program (FP7/2013-2016) for the Fuel Cell and Hydrogen Joint Technology Initiative under grant agreement CATAPULT $n^{\circ} 0.325268$ is gratefully acknowledged. This work was supported by the German Research Foundation (DFG) and the Technical University of Munich (TUM) in the framework of the Open Access Publishing Program.

\section{Author contributions}

H.E. and H.G. proposed the idea and outline of the research. J.S. designed and performed the experiments and P.L.T. carried the XPS experiments and data analysis. J.S. wrote the manuscript under the supervision of H.E. and H.G.

\section{Competing interests}

The authors declare no competing interests.

\section{Additional information}

Correspondence and requests for materials should be addressed to H.A.E.-S.

Reprints and permissions information is available at www.nature.com/reprints.

Publisher's note Springer Nature remains neutral with regard to jurisdictional claims in published maps and institutional affiliations.

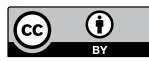

Open Access This article is licensed under a Creative Commons Attribution 4.0 International License, which permits use, sharing, adaptation, distribution and reproduction in any medium or format, as long as you give appropriate credit to the original author(s) and the source, provide a link to the Creative Commons license, and indicate if changes were made. The images or other third party material in this article are included in the article's Creative Commons license, unless indicated otherwise in a credit line to the material. If material is not included in the article's Creative Commons license and your intended use is not permitted by statutory regulation or exceeds the permitted use, you will need to obtain permission directly from the copyright holder. To view a copy of this license, visit http://creativecommons.org/licenses/by/4.0/.

(c) The Author(s) 2020 